\title{
ARTICLE
}

\section{A response function methodology to calculate induced fission neutron flux distribution in an active interrogation system}

\author{
William Walters $^{\mathrm{a}^{*}}$, Alireza Haghighat ${ }^{\mathrm{a}}$, Katherine Royston ${ }^{\mathrm{a}}, \mathrm{Ce} \mathrm{Yi}^{\mathrm{b}}$ and Glenn Sjoden ${ }^{\mathrm{b}}$ \\ ${ }^{a}$ Nuclear Engineering Program, VTRC, Virginia Tech, Arlington, Virginia, USA; ${ }^{b}$ Nuclear and Radiological Engineering Program, \\ Georgia Tech, Atlanta, Georgia, USA
}

\begin{abstract}
A hybrid deterministic and Monte Carlo radiation transport methodology is being developed for modeling an active interrogation system for detection of special nuclear material (SNM) in a container. This methodology includes four steps: i) ( $n, \gamma)$ interaction and subcritical multiplication in cargo; ii) $(n, \gamma)$ interaction due to fission neutrons throughout the cargo; iii) gamma transport to the detector window; iv) detection of gamma-rays by the detector. This methodology enables accurate determination of neutron/gamma fluxes in real times for comparison with measurements, and examination of various materials compositions. In this paper, we will address the first step of the methodology. We have developed a response-function formulation to calculate the subcritical multiplication and the neutron flux distribution. Using the MCNP Monte Carlo code, the response coefficients are pre-calculated for various cargo materials and SNM combinations. We have demonstrated that the new response function methodology yields fission neutron rates which are in excellent agreement with the full Monte Carlo calculation predictions (within $10 \%$ for all sampled locations including those near the wall), while achieving significant speedups of several orders of magnitude.
\end{abstract}

Keywords: active interrogation; Monte Carlo

\section{Introduction}

A hybrid deterministic and Monte Carlo radiation transport methodology is being developed for modeling an active interrogation system for detection of Special Nuclear Materials (SNMs) in a cargo container. Due to the enormous quantity of cargo being transported, real-time simulation with standard particle-transport techniques is impractical, and therefore a new fast technique is required.

In this paper, we will address the calculation of the fission rate in the SNM. We have developed new formulations based on the response function methodology $[1,2]$ for determination of fission neutron density due to subcritical multiplication of the SNM in the cargo. For this paper, highly enriched uranium (HEU) is used as the SNM.

The accuracy and performance of the new formulations are determined by comparison with calculations using the Monte Carlo MCNP5 code [3].

The paper is organized as follows. Section 2 derives the response function formulation for subcritical multiplication. Section 3 discusses the reference model used, Section 4 outlines the calculation of the response coefficients, Section 5 gives results of the response

*Corresponding author. Email: waltersw@vt.edu method as compared to MCNP and Section 6 analyzes the computation time of the two methods.

\section{Derivation of response-function formulation for subcritical multiplication}

This formulation is developed by splitting the cargo container into two regions: the cargo region and the HEU region, or region-of-interest (ROI). Calculations are performed on each individually to determine response coefficients, and then these are combined to calculate the total fission rate in the HEU. The aim of the method is to greatly reduce the number of variables in solving the problem. In this implementation, we collapse the problem into only four variables:

- $S$ : external source

- $J_{\text {in, } \mathrm{A}}$ total integrated (over energy and area) current entering ROI

- $J_{\text {out }, \mathrm{A}}$ : total integrated current exiting ROI

- $F$ : fission rate in ROI

These variables are shown in Figure 1.

The goal of the method is: given the external source $S$, calculate the fission rate $F$ inside the ROI. To accomplish this, the problem model is split up into several smaller, de-coupled models. Coefficients are then calculated to link the different variables. In the models shown here, a black region represents a region 


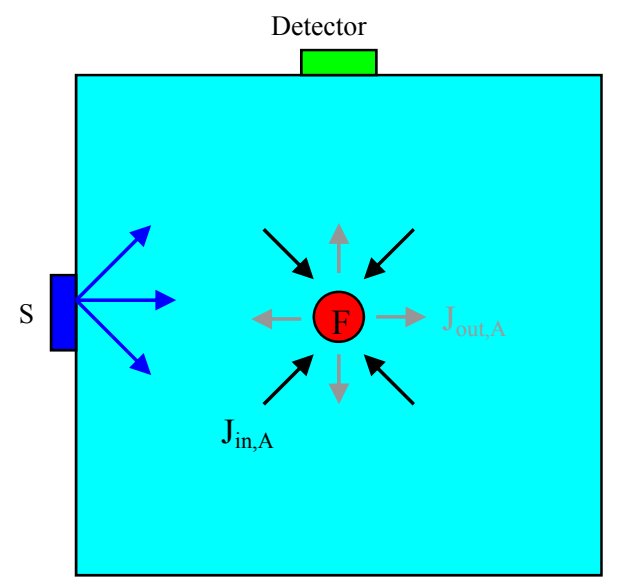

Figure 1. Cargo model for the response method. The cargo is in light blue, the region of interest is in red, source is blue and the detector is in green.

that is not modeled (i.e., neutron importance is set to 0 ). Only one region is modeled at a time, allowed for the complete de-coupling of each model. In order to couple the problems together, we used the surface source write/read capability of MCNP. A surface source is written in the first model, which is read in as the source for the second model, etc. This surface source contains all the information about particles crossing, such as position, energy and direction.

The goal of the first model is: from the source $S$, calculate the current into the ROI, $J_{\text {in, } \mathrm{A}}$, as shown below. Then we can calculate the coefficient relating these two as in Eq. (1). A model for this calculation is shown in Figure 2.

$$
\alpha_{s, i n}=J_{i n} / S
$$

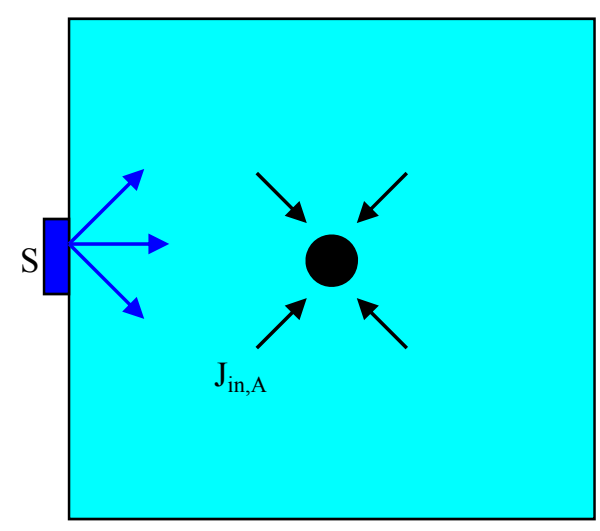

Figure 2. Model for calculation of $\alpha_{\mathrm{s}, \mathrm{in}}$.

Next, from the current going into the ROI, we calculate the current leaving the ROI as in Eq. (2). From this current, we can also calculate the fission source in the ROI, as in Eq. (3). The model for this is shown in Figure 3.

$$
\begin{aligned}
& \alpha_{\text {in out }}=J_{\text {out }} / J_{\text {in }} \\
& \alpha_{\text {in }, f}=f / J_{\text {in }}
\end{aligned}
$$

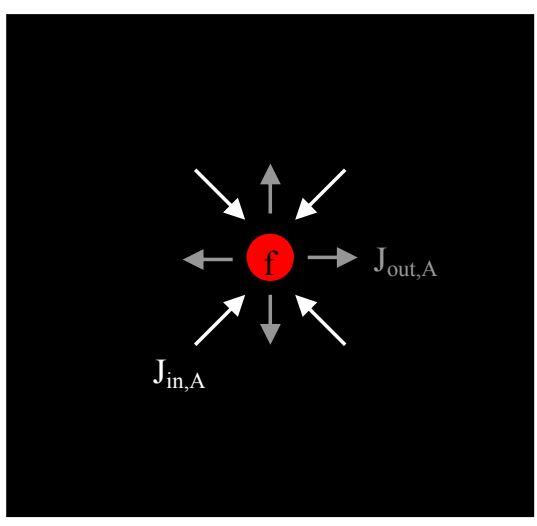

Figure 3. Model for calculation of $\alpha_{\text {in,out }}$ and $\alpha_{\text {in,f }}$.

Finally, from the current leaving the ROI we now calculate the current returning to the ROI (i.e., reflection), as in Eq. (4), and shown in Figure 4. From here, we definte the new currents $\mathrm{J}{ }_{\text {in }}$ and $\mathrm{J}{ }_{\text {out }}$ as being different from $\mathrm{J}_{\text {in }}$ and $\mathrm{J}_{\text {out }} . \mathrm{J}^{\prime}$ in and $\mathrm{J}^{\prime}$ out (the new currents) will have different energy spectra (one being driven by the external source spectrum vs. being driven by the fission spectrum). This will result in the coefficients for each being slightly different.

$$
\alpha_{\text {out }, \text { in }}=J_{\text {in }}^{\prime} / J_{\text {out }}
$$

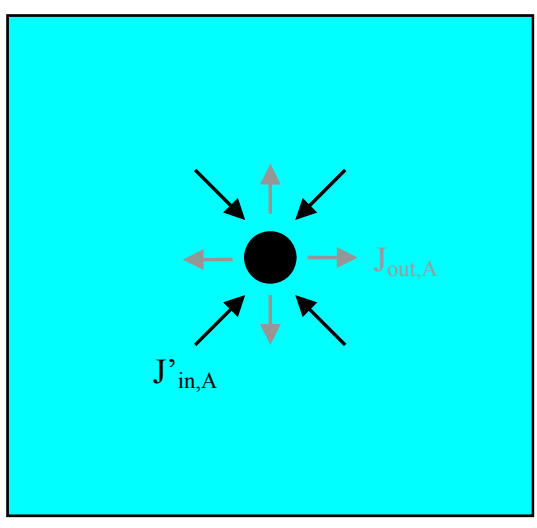

Figure 4. Model for calculation of $\alpha_{\text {out,in }}$.

These previous calculations are repeated using the new incoming/outgoing spectra to yield new coefficients as in Eqs. (5)-(7)

$$
\begin{aligned}
& \alpha_{\text {in out }}^{\prime}=J_{\text {out }}^{\prime} / J^{\prime}{ }_{\text {in }} \\
& \alpha_{\text {in, } f}^{\prime}=f^{\prime} / J^{\prime}{ }_{\text {in }} \\
& \alpha_{\text {out ,in }}^{\prime}=J^{\prime}{ }_{\text {in }} / J^{\prime}{ }_{\text {out }}
\end{aligned}
$$

Once we have these coefficients, we can calculate all of variables in Eqs. (8)-(12), given the source strength $S$.

$$
\begin{aligned}
& J_{\text {in }}=S \alpha_{s, \text { in }} \\
& J_{\text {out }}=J_{\text {in }} \alpha_{\text {in }, \text { out }}
\end{aligned}
$$




$$
\begin{aligned}
& J_{\text {in }}{ }^{\prime}=J_{\text {out }} \alpha_{\text {out }, \text { in }}+J_{\text {out }}{ }^{\prime} \alpha_{\text {out }, \text { in }}^{\prime} \\
& J_{\text {out }}{ }^{\prime}=J_{\text {in }}{ }^{\prime} \alpha_{\text {in out }}^{\prime} \\
& F=J_{\text {in }} \alpha_{\text {in }, f}+J_{\text {in }}{ }^{\prime} \alpha_{\text {in }, f}^{\prime}
\end{aligned}
$$

Or, solving for the total fission rate $F$, we have:

$$
F=S \alpha_{s, i n}\left(\alpha_{i n, f}+\frac{\alpha_{i n, f}^{\prime} \alpha_{i n, o u t} \alpha_{\text {out }, \text { in }}}{1-\alpha_{\text {in,out }}^{\prime} \alpha_{\text {out }, \text { in }}^{\prime}}\right)
$$

\section{Description of reference model}

To demonstrate the methodology, we consider a cargo container, which is inspected using a detector-source assembly as shown in Figure 5.

The neutron source is a D-T source with energy of 14.1 MeV. Not shown in the diagram is the SNM material, i.e., HEU, which is placed at the center of container as a sphere of radius $6.75 \mathrm{~cm}$. This size sphere corresponds to $25 \mathrm{~kg}$ of HEU. The neutron source is represented as a surface source of size $13.5 \times 13.5 \mathrm{~cm}^{2}$, and the detector window is represented as a surface of $13.5 \times 13.5 \mathrm{~cm}^{2}$.

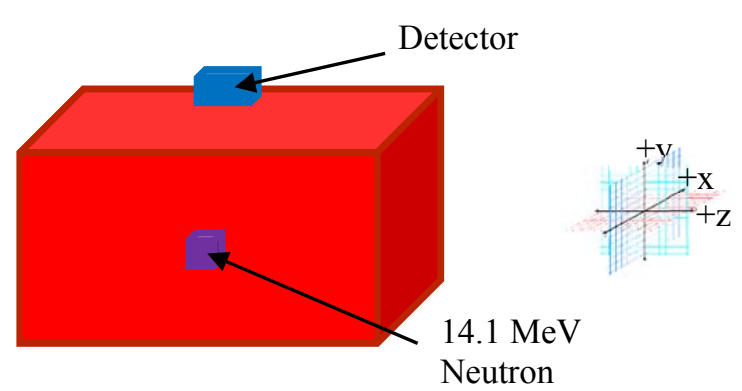

Figure 5. Cargo container reference model.

The size of the reference container is $8.5^{\prime}(2.6 \mathrm{~m}) \times 8.5^{\prime}$ $(2.6 \mathrm{~m}) \times 20^{\prime}(6.1 \mathrm{~m})$. Considering that the HEU sphere is placed at the center of the container, then we may reduce the model size by considering the geometric symmetry along the $\mathrm{z}$-axis (container depth). For this model, the container is filled with water of one-third nominal density (chosen as a proxy for a generic hydrogenous cargo material). The container is made of stainless steel of $1 \mathrm{~cm}$ thickness, and the D-T source is an isotropic surface source.

\section{Determination of response coefficients}

By performing a series of fixed-source MCNP Monte Carlo calculations, we determine the response coefficients of Eqs. (1)-(7).

Table 1 gives the response coefficients for $0.33 \mathrm{~g} / \mathrm{cc}$ water.

Since a cargo container is very large compared to the ROI, and ROI generally should be away from the container wall, as it would make for easy detection by
Table 1. Response coefficients for $0.33 \mathrm{~g} / \mathrm{cc}$ water.

\begin{tabular}{|c|c|}
\hline Coefficient & Value \\
\hline$\alpha_{\text {s,in }}$ & $4.233 \mathrm{E}-04$ \\
$\alpha_{\text {in,out }}$ & 4.457 \\
$\alpha_{\text {out,in }}$ & 0.149 \\
$\alpha_{\text {in,out }}^{\prime}$ & 3.474 \\
$\alpha_{\text {in,f }}$ & 5.944 \\
$\alpha_{\text {in,f }}^{\prime}$ & 4.515 \\
$\alpha_{\text {out,in }}^{\prime}$ & 0.158 \\
\hline
\end{tabular}

standard passive methods, the only coefficient that should change substantially is the source-ROI coupling, while the other coefficients remain relatively constant. Therefore, in the following subsection, we have developed a simple formulation for determination of $\alpha_{\mathrm{s}, \text { in }}$ as a function of the distance from the source $(r)$.

\subsection{Determination of response coefficients as function of ROI position}

Considering the large size of the cargo container, we have demonstrated that the $\alpha_{s, i n}(r)$ ( $\mathrm{r}$ being the source-ROI distance) can be determined by using the formulation of the dispersion of a point source in a diffusive medium, as in Eq. (14).

$$
\alpha_{s, i n}=\frac{\alpha_{0}}{r} \exp \left(-\sigma_{0} r\right)
$$

For data, we obtained the source-ROI coefficient for 6 distances from $40-140 \mathrm{~cm}$. The parameters $\alpha_{0}$ and $\sigma_{0}$ were estimated using the non-linear least squares method. The point at $40 \mathrm{~cm}$ was left out of the regression since it is too close for the diffusive approximation to be valid. The coefficient values and values of the fit are shown in Figure 6.

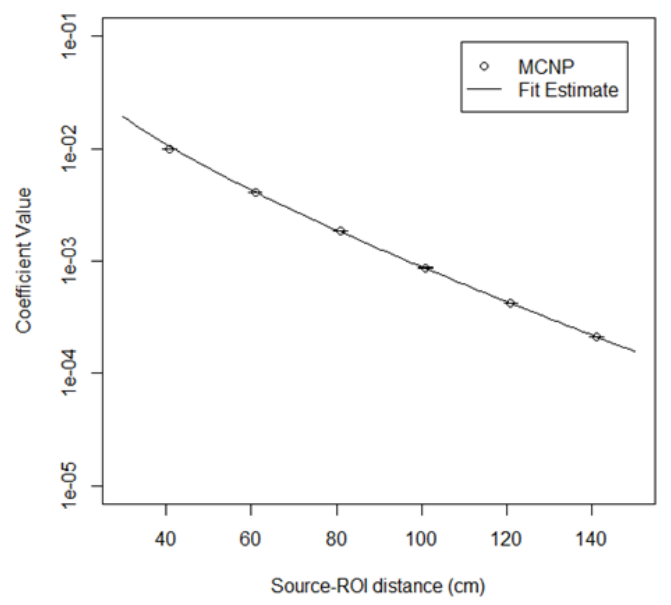

Figure 6. Source-ROI coefficients as a function of distance.

\section{Results}

In order to test the accuracy of the methodology, we examine a series of full MCNP calculations and response method calculations for a series of possible 
ROI locations. The ROI locations tested are shown in Figure 7.

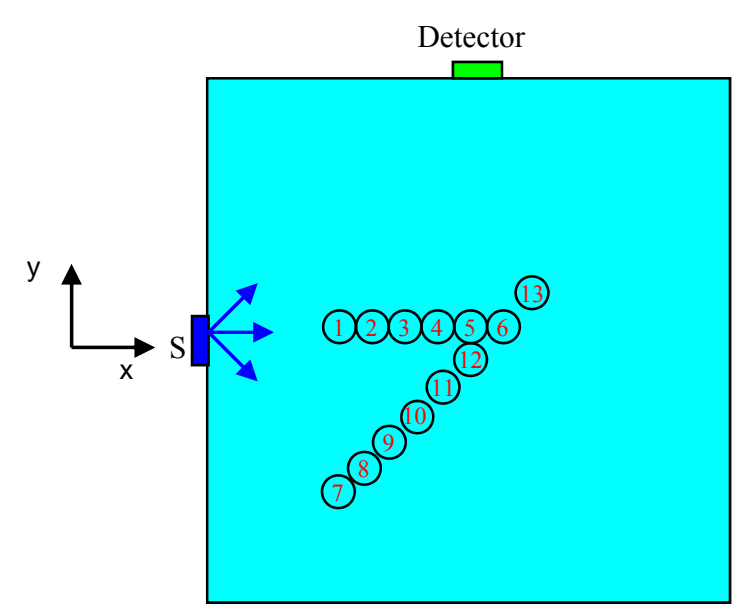

Figure 7. ROI test locations.

Table 2. ROI test locations.

\begin{tabular}{|c|c|c|c|}
\hline ROI Location & $\mathrm{x}(\mathrm{cm})$ & $\mathrm{y}(\mathrm{cm})$ & $\begin{array}{c}\text { Total } \\
\text { distance }(\mathrm{cm})\end{array}$ \\
\hline 1 & 40.92 & 0 & 40.92 \\
2 & 60.92 & 0 & 60.92 \\
3 & 80.92 & 0 & 80.92 \\
4 & 100.92 & 0 & 100.92 \\
5 & 120.92 & 0 & 120.92 \\
6 & 140.92 & 0 & 140.92 \\
7 & 40.92 & 80 & 89.89 \\
8 & 60.92 & 60 & 85.56 \\
9 & 80.92 & 40 & 90.34 \\
10 & 90.92 & 30 & 95.82 \\
11 & 100.02 & 20 & 102.96 \\
12 & 110.92 & 10 & 111.45 \\
13 & 130.92 & -10 & 131.38 \\
\hline
\end{tabular}

Table 2 gives the $(\mathrm{x}, \mathrm{y})$ positions for these locations, which are all located at $\mathrm{z}=0$.

Table 3 compares the results of response function methodology with the Monte Carlo predictions. This indicates that the result of the new response function formulation is in excellent agreement with the Monte Carlo prediction. If the HEU sphere comes very close to the cargo wall (as in location 7), the error goes up some but only slightly $(\sim 10 \%)$. This is from the coefficients being calculated only for the center of the cargo. Again, it is reasonable to expect that any HEU would be hidden far from the edge of the cargo container, so this is not a large problem. It should also be noted that the fission rate is changing by two orders of magnitude, so a $10 \%$ difference is not especially large.
Table 3. MCNP vs response method to calculate fission density for various ROI locations.

\begin{tabular}{|c|c|c|c|c|}
\hline $\begin{array}{c}\text { ROI } \\
\text { Location }\end{array}$ & MCNP & $\begin{array}{c}\text { Uncert. } \\
(1-\sigma)\end{array}$ & Estimate & Difference \\
\hline 1 & $1.40 \mathrm{E}-01$ & $0.46 \%$ & $1.33 \mathrm{E}-01$ & $-5.22 \%$ \\
2 & $5.50 \mathrm{E}-02$ & $0.64 \%$ & $5.23 \mathrm{E}-02$ & $-5.00 \%$ \\
3 & $2.39 \mathrm{E}-02$ & $0.90 \%$ & $2.31 \mathrm{E}-02$ & $-3.25 \%$ \\
4 & $1.11 \mathrm{E}-02$ & $0.45 \%$ & $1.09 \mathrm{E}-02$ & $-1.91 \%$ \\
5 & $5.32 \mathrm{E}-03$ & $0.40 \%$ & $5.31 \mathrm{E}-03$ & $-0.21 \%$ \\
6 & $2.60 \mathrm{E}-03$ & $0.52 \%$ & $2.67 \mathrm{E}-03$ & $2.55 \%$ \\
7 & $1.48 \mathrm{E}-02$ & $1.31 \%$ & $1.64 \mathrm{E}-02$ & $10.18 \%$ \\
8 & $1.92 \mathrm{E}-02$ & $1.58 \%$ & $1.93 \mathrm{E}-02$ & $0.44 \%$ \\
9 & $1.63 \mathrm{E}-02$ & $1.37 \%$ & $1.61 \mathrm{E}-02$ & $-1.64 \%$ \\
10 & $1.37 \mathrm{E}-02$ & $1.48 \%$ & $1.31 \mathrm{E}-02$ & $-4.18 \%$ \\
11 & $1.04 \mathrm{E}-02$ & $1.55 \%$ & $1.01 \mathrm{E}-02$ & $-2.91 \%$ \\
12 & $7.43 \mathrm{E}-03$ & $1.60 \%$ & $7.42 \mathrm{E}-03$ & $-0.24 \%$ \\
13 & $3.69 \mathrm{E}-03$ & $1.80 \%$ & $3.70 \mathrm{E}-03$ & $0.08 \%$ \\
\hline
\end{tabular}

\section{Computation time}

Using the response method, almost all of the computation time is taking performing the series of pre-calculations that lead to the response coefficients. Table 4 gives the computation times for determination of response coefficients.

Table 4. Response method pre-calculation computation times.

\begin{tabular}{|c|c|c|}
\hline Coefficient & $\begin{array}{c}\text { Relative Error } \\
(1-\text { sigma })\end{array}$ & Computation Time \\
\hline$\alpha_{\text {s,in }}$ & All $<0.5 \%$ & 360 min $(6$ locations) \\
$\alpha_{\text {in,out }}$ & $0.40 \%$ & $\begin{array}{c}10 \text { min (both source } \\
\text { spectra) }\end{array}$ \\
$\alpha_{\text {out,in }}$ & $0.50 \%$ & $\begin{array}{c}120 \text { min ( both source } \\
\text { spectra ) }\end{array}$ \\
\hline \multicolumn{2}{|c|}{ Total Time } & $\mathbf{4 9 0 ~} \mathbf{m i n}$ \\
\hline
\end{tabular}

However, after these pre-calculations, any subsequent calculations take almost no time at all, especially when compared to a straight MCNP calculation, as in Table 5.

Table 5. Subsequent computation times.

\begin{tabular}{|l|c|}
\hline Straight MCNP & $350 \mathrm{~min}$ \\
\hline Response Method Calculation & $<<<1 \mathrm{~s}$ \\
\hline
\end{tabular}

Clearly, the response function method is orders of magnitude faster than straight MCNP, if even only a few calculations need to be performed.

\section{Conclusion}

We have developed a successful response-function method to calculate the induced fission in an active interrogation system. The response method achieves excellent accuracy as compared to Monte Carlo, with most test cases within 5\%. Even for cases very close to 
the wall where large errors would be expected, the difference rises to only $10 \%$. At the same time, we achieved a speedup of several orders of magnitude.

\section{Acknowledgements}

The authors wish to thank the U.S. National Nuclear Security Administration (NNSA) for the funding of this project.

\section{References}

[1] G. I. Bell and S. Glasstone, Nuclear Reactor Theory, Robert E. Krieger Publishing, Malabar, FL, (1979).

[2] D. Zhang and F. Rahnema, An efficient hybrid stochastic/deterministic coarse mesh neutron transport method, Annals of Nuclear Energy 41 (2012), pp. 1-11.

[3] X-5 Monte Carlo Team, MCNP-A General Monte Carlo Code for Neutron and Photon Transport, Version 5, Los Alamos National Laboratory, (2003). 